\title{
Ten-Year Trends in Antiemetic Prescribing in Patients Receiving Highly Emetogenic Chemotherapy
}

\author{
Ciara C. O'Sullivan, MB BCh ${ }^{\mathrm{a}}$; Holly K. Van Houten, BA ${ }^{\mathrm{b}, \mathrm{c}}$; Lindsey R. Sangaralingham, MPH ${ }^{\mathrm{b}, \mathrm{c}}$; \\ Alexis D. Leal, MDa; Shivani Shinde, $\mathrm{MD}^{\mathrm{a}}$; Hongfang Liu, $\mathrm{PhD}^{\mathrm{d}}$; David Ettinger, MD; \\ Charles L. Loprinzi, MDa; and Kathryn J. Ruddy, MD, MPH ${ }^{a}$
}

\begin{abstract}
Purpose: Prevention of chemotherapy-induced nausea and vomiting is essential to preserve quality of life in patients with cancer receiving highly emetogenic chemotherapy (HEC). Recently, new drugs (eg, fosaprepitant, and the newer neurokinin-1 receptor antagonists [NK1RAs] rolapitant and netupitant) and updated antiemetic guidelines have emerged. However, trends in real-world antiemetic use are understudied. Methods: We identified patients treated with an initial dose of HEC (either cisplatin or doxorubicin/cyclophosphamide) from January 2006 to June 2016 using administrative claims data from a US commercial insurance database (OptumLabs). Antiemetic use was determined by identifying intravenous/oral/transdermal administration within \pm 1 day of the chemotherapy dose and/or prescription fill from 14 days before to 7 days after chemotherapy. We used descriptive statistics to present patient demographics, chemotherapy drugs administered, presence/absence of a central intravenous access device, and antiemetics used. Results: A total of 23,030 patients (67.3\%) received doxorubicin/cyclophosphamide and 11,206 (32.7\%) received cisplatin. Dexamethasone and 5-hydroxytryptamine 3 receptor antagonists (5-HT3RAs) were consistently used by $85 \%$ to $95 \%$ of patients, consistent with guideline recommendations. NK1RAs were underused early on, but use increased to approximately $80 \%$ in the most recently evaluated year. Fosaprepitant use increased precipitously starting in 2009, preceding a sharp decrease in aprepitant use beginning in 2011. Receipt of olanzapine, rolapitant, and netupitant was minimal throughout the study period. Conclusions: Dexamethasone and 5-HT3RAs were used by most patients receiving HEC, in accordance with guideline recommendations. NK1RA use was less adherent with guidelines.
\end{abstract}

J Natl Compr Canc Netw 2018;16(3):294-299 doi: 10.6004 ljnccn.2017.7043

Chemotherapy-induced nausea and vomiting (CINV) is common during cancer treatment due to a complex multifactorial process involving several different pathways and transmitters. Highly emetogenic chemotherapy (HEC) regimens are associated with $>90 \%$ risk of emesis if no antiemetics are provided. ${ }^{1}$ Delayed nausea and vomiting is a particular problem with HEC because it is often underestimated by nurses and physicians. ${ }^{2}$ Effective prevention is important for maintaining quality of life. ${ }^{3,4}$ Significant advances in the management of CINV (eg, availability of 5-hydroxytryptamine 3 receptor antagonists [5-HT3RAs], the neurokinin-1 receptor antago-

From a Department of Oncology, and ${ }^{b}$ Robert D. and Patricia E. Kern Center for the Science of Health Care Delivery, Mayo Clinic, Rochester, Minnesota; 'OptumLabs, Cambridge, Massachusetts; ${ }^{d}$ Department of Health Sciences Research, Mayo Clinic, Rochester, Minnesota; and eThe Sidney Kimmel Comprehensive Cancer Center at Johns Hopkins, Baltimore, Maryland.

Submitted August 10, 2017; accepted for publication September 29, 2017. nists [NK1RAs], and olanzapine) have occurred over the past 2 decades. ${ }^{5}$ FDA approval of 5-HT3RAs in the late 1990s was an important step toward improved control of CINV. Ondansetron and granisetron are first-generation 5-HT3RAs, and palonosetron is a second-generation 5-HT3RA that has a significantly longer half-life than the first-generation compounds. The combination of palonosetron and glucocorticoids has resulted in superior control of delayed emesis compared with glucocorticoids combined with first-generation 5-HT3RAs., ${ }^{6,7}$ The subsequent approval of the NK1RAs (initially oral aprepitant [2003]) was another landmark advance in antiemetic

The authors have disclosed that they have no financial interests, arrangements, affiliations, or commercial interests with the manufacturers of any products discussed in this article or their competitors.

Correspondence: Kathryn J. Ruddy, MD, MPH, Department of Oncology, Mayo Clinic, 200 1st Street SW, Rochester, MN 55905.

E-mail: Ruddy.Kathryn@mayo.edu 
Trends in Antiemetic Prescribing

prophylaxis. Aprepitant was shown to consistently prevent CINV across a broad range of chemotherapy regimens. ${ }^{8,9}$ Fosaprepitant is a water-soluble derivative of aprepitant and is rapidly converted to aprepitant after intravenous administration. A large randomized noninferiority trial demonstrated that a single dose of intravenous fosaprepitant on day 1 of chemotherapy was comparable in efficacy to the standard 3-day oral aprepitant regimen. ${ }^{10}$ The more convenient intravenous dosing schedule was offered as a way to assure medication compliance. ${ }^{11}$

In the recent past, several groups reported that fosaprepitant caused substantial venous irritation, especially with doxorubicin-based chemotherapy regimens that were administered by peripheral venous access. ${ }^{12-15}$ For example, venous toxicity was observed in 35\% of patients receiving intravenous fosaprepitant followed by doxorubicin/cyclophosphamide (AC) compared with only $2 \%$ of patients receiving oral aprepitant with AC. ${ }^{16}$ In a separate retrospective study, only $7 \%$ of patients receiving fosaprepitant with nonanthracycline platinum-based chemotherapy regimens experienced venous toxicity. ${ }^{13}$

A variety of antiemetic guidelines are available from $\mathrm{NCCN}, \mathrm{ASCO}, \mathrm{ESMO}$, and the Multinational Association of Supportive Care in Cancer (MASCC). ${ }^{17-19}$ The current consensus is that a combination of dexamethasone, a 5-HT3RA (eg, ondansetron, granisetron, dolasetron, or palonosetron), and an NK1RA (eg, aprepitant, fosaprepitant, netupitant, or rolapitant) is an appropriate preventative regimen in patients receiving HEC. However, considerable debate remains regarding how long patients should receive dexamethasone after chemotherapy. ${ }^{20}$ Recent NCCN Clinical Practice Guidelines in Oncology (NCCN Guidelines) for Antiemesis suggest that olanzapine be considered either in place of or in addition to the NK1RA in patients receiving HEC. ${ }^{19}$

Extensive literature exists on the use of antiemetics in patients with cancer. For example, a PubMed search using the terms "antiemetics" and "cancer" identified 16,392 potentially relevant articles. Further, there are multiple options in the ASCO and ESMO/MASCC guidelines pertaining to the use of 5-HT3RAs and NK1RAs, ${ }^{1,18}$ which vary in modes of administration (oral, intravenous, or transdermal) and cost. The current NCCN Guidelines for Antiemesis illustrate the 42 different "guideline-approved" options for the prevention of acute and delayed emesis in patients with cancer receiving $\mathrm{HEC}$ (available at NCCN.org [page AE-5]). ${ }^{19}$ These include various combinations of several different agents, schedules, and modes of administration.

The current study was developed to address a number of questions regarding the use of antiemetics in the real clinical-practice world, including how often dexamethasone is really used in patients receiving HEC, which of the 5HT3RAs and NK1RAs have been used over time, and whether published data regarding vein toxicity from fosaprepitant change the its use, particularly in patients who received peripheral vein drug administration. Additionally, given pilot nonrandomized data dating back to $2004^{21,22}$ and pilot randomized controlled data from 2011 supporting that olanzapine was helpful for decreasing CINV, ${ }^{23}$ we also sought to determine whether olanzapine use increased in clinical practice before the 2016 publication of results of a doubleblind, randomized, placebo-controlled clinical trial. ${ }^{24}$

\section{Methods}

\section{Data Source}

We performed a retrospective study using the OptumLabs Data Warehouse, which includes administrative claims data for $>100$ million privately insured and Medicare Advantage enrollees in the United States over the past 20 years. ${ }^{25}$ The database contains longitudinal health information from geographically diverse regions across the United States, with greatest representation from the South and Midwest. ${ }^{26}$ The included health plans provide data covering several domains, including enrollee information (insurance plan, sex, age, race/ethnicity, region of residence, dates of eligibility), outpatient pharmacy claims (prescribing physician, fill dates, days of supply, strength), professional claims (eg, visits to physicians' offices), and facility claims (eg, hospitalizations). ${ }^{26}$ This study analyzed preexisting, deidentified data, and was therefore deemed exempt from Institutional Review Board approval.

\section{Study Population}

We identified patients who initiated AC, TAC (paclitaxel or docetaxel with doxorubicin and cyclophosphamide), or cisplatin between January 2006 and March 2016. Subsequent cisplatin doses had to be at least 14 days later than the first dose to reduce the likelihood that patients receiving low-dose weekly (less emetogenic) cisplatin were included in 
the analysis. Patients were required to have at least 30 days of medical and pharmacy insurance coverage prior to and after initial chemotherapy treatment. Antiemetic use was determined by identifying intravenous/oral/transdermal administration within \pm 1 day of index chemotherapy and/or prescription fill within 14 days prior to and 7 days after index chemotherapy. Antiemetic medications evaluated included dexamethasone, ondansetron, granisetron, palonosetron, aprepitant, fosaprepitant, netupitant, rolapitant, and olanzapine.

\section{Statistical Analysis}

Patient characteristics (age, sex, race, region) were described using mean (SD) or frequencies (percentage) as appropriate. The proportion of patients initiating AC, TAC, or cisplatin chemotherapy regimens (denominator) and taking an antiemetic medication (numerator) in each quarter of the study period was calculated. Trends of antiemetic use were presented overall and separately for 5HT3RAs and NK1RAs. Statistical analysis using SAS 9.4 (SAS Institute Inc., Cary, NC) was used to evaluate trends in antiemetic use over time.

\section{Results}

We identified 34,236 patients who received AC, TAC, or cisplatin from the first quarter of 2006 (Q1) to the second quarter (Q2) of 2016 (Figure 1). Patient demographics are shown in Table 1.

Figure 2 shows overall trends in antiemetic prescribing from 2006 through 2016. Predictably, nearly all patients who received HEC were given antiemetics. Dexamethasone use was most common and consistent over time, identified in $85 \%$ to $90 \%$ of patients consistently over the entire study period.

\section{Trends in Serotonin Receptor Antagonist Use}

Figure 3, which focuses on the use of 5HT3RAs, illustrates that the proportion of patients who received ondansetron increased from approximately $33 \%$ in 2006 to $63 \%$ in 2016 . The proportion of patients who received palonosetron also increased over time (from $55 \%$ in 2006 to $71 \%$ in 2016), whereas the proportion who received granisetron decreased (from approximately $17 \%$ in 2006 to $<10 \%$ in 2016). Dolasetron use also decreased over time, from approximately $10 \%$ in 2006 to $<1 \%$ in 2016 , with the

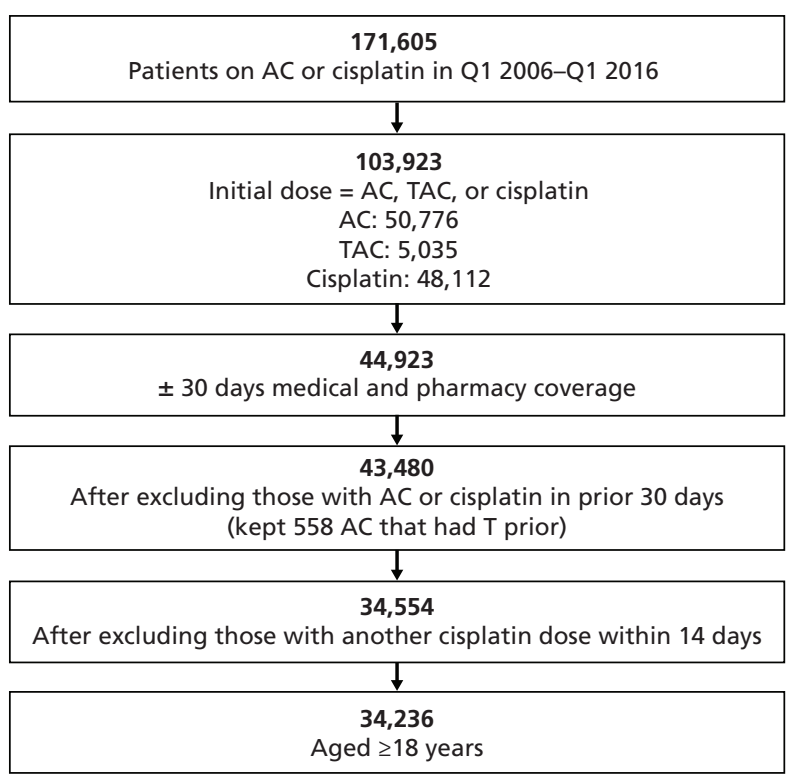

Figure 1. CONSORT diagram.

Abbreviations: AC, doxorubicin and cyclophosphamide; Q1, first quarter; T, docetaxel; TAC, paclitaxel or docetaxel with doxorubicin and cyclophosphamide.

most noticeable decline occurring between 2010 and 2011 (from $\approx 5 \%$ to $<1 \%$ ).

\section{Trends in NK1RA Use}

Figure 4 illustrates that aprepitant, which was the only NK1RA clinically available prior to 2008, was used in $40 \%$ to $50 \%$ of patients around that time, but then its use decreased sharply between 2010 and 2011, declining to $<5 \%$ in 2016 . This decline in aprepitant use coincided with a dramatic increase in the use of fosaprepitant after it became available in 2008. In addition to fosaprepitant replacing the use of aprepitant, its availability appeared to increase the use of an NK1RA for HEC from approximately $49 \%$ to approximately $80 \%$. No evidence suggested that the underuse of NK1RA (vs dexamethasone and 5HT3RA) resulted from physicians believing that they did not need it when they used the more effective 5HT3RA (palonosetron), because the use of an NK1RA was slightly more likely (nonsignificantly) in patients who received palonosetron compared with another 5HT3RA.

Notably, no decrease in use of fosaprepitant was observed after initial reports in 2014 that it may cause venous toxicity. Understanding that the intravenous toxicity of fosaprepitant only seemed to occur in patients with peripheral vein administration, the use of aprepitant and fosaprepitant were evaluated in patients 


\begin{tabular}{|c|c|}
\hline Characteristic & $\begin{array}{c}n(\%) \\
(N=34,236)\end{array}$ \\
\hline Median age, y & 58 \\
\hline \multicolumn{2}{|l|}{ Age group } \\
\hline $18-34 y$ & $1,227(4 \%)$ \\
\hline $35-54$ y & $12,294(36 \%)$ \\
\hline $55-64$ y & $10,201(30 \%)$ \\
\hline$\geq 65 y$ & $10,514(31 \%)$ \\
\hline \multicolumn{2}{|l|}{ Sex } \\
\hline Female & $23,392(69 \%)$ \\
\hline Male & $10,844(32 \%)$ \\
\hline \multicolumn{2}{|l|}{ Region } \\
\hline Midwest & $10,559(31 \%)$ \\
\hline Northeast & $4,477(13 \%)$ \\
\hline South & $14,959(44 \%)$ \\
\hline West & $4,241(12 \%)$ \\
\hline \multicolumn{2}{|l|}{ Race } \\
\hline Asian & $874(3 \%)$ \\
\hline Black & $4,031(12 \%)$ \\
\hline Hispanic & $2,345(7 \%)$ \\
\hline Unknown & $1,994(6 \%)$ \\
\hline White & $24,992(73 \%)$ \\
\hline \multicolumn{2}{|c|}{ Chemotherapy regimen } \\
\hline$A C$ & $21,066(62 \%)$ \\
\hline TAC & $1,964(6 \%)$ \\
\hline Cisplatin only & $11,206(33 \%)$ \\
\hline
\end{tabular}

Abbreviations: AC, doxorubicin and cyclophosphamide; TAC, paclitaxel or docetaxel with doxorubicin and cyclophosphamide.

with only peripheral vein access, excluding those with central vein access. These data similarly revealed that there was not any apparent decrease in fosaprepitant use after 2014 in patients with only peripheral vein access. During the dates assessed in the current project, uptake of netupitant/palonosetron and rolapitant was low, with only minimal use in 2015-2016 (Figure 2B).

\section{Olanzapine Use}

Use of olanzapine was minimal over time, but started to slowly increase in the third quarter of 2015 (Figure 2).

\section{Subsets of Subjects Receiving Different Chemotherapy Regimens}

Trends in antiemetic use in patients who received AC, TAC, or cisplatin were similar to those observed in the overall study population.
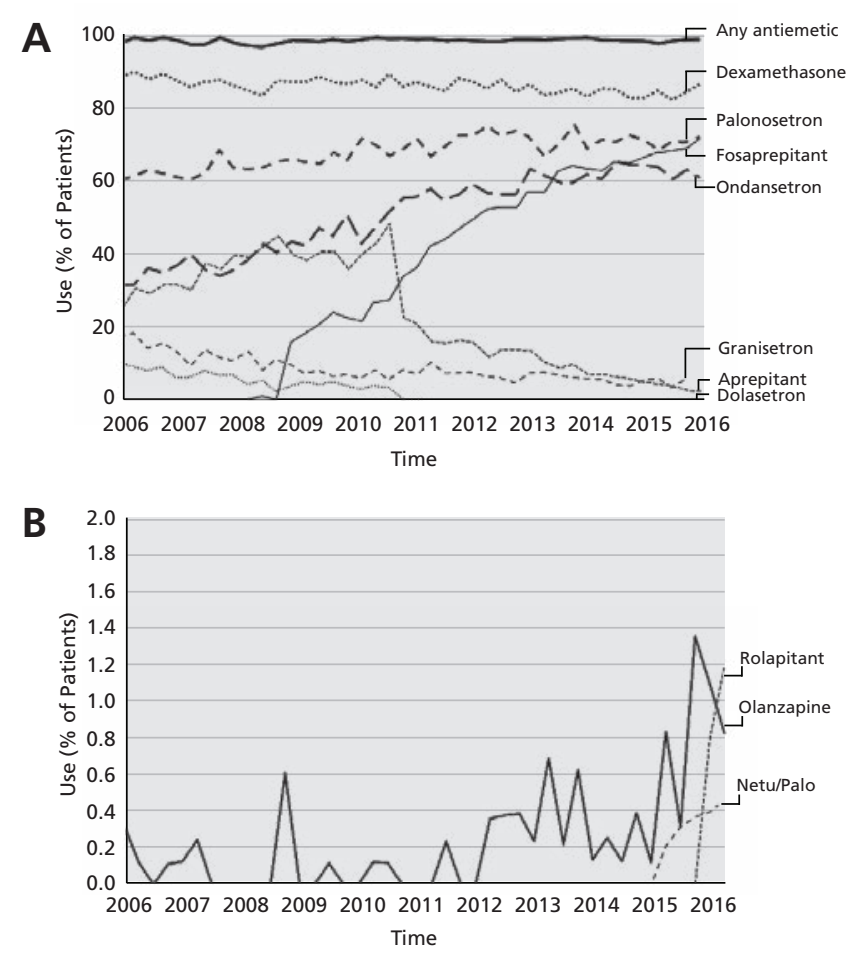

Figure 2. (A) Overall trends in the use of antiemetic agents from 2006 through 2016, and (B) expansion of the $0 \%-2 \%$ data to illustrate the minimal use of rolapitant, netupitant (netu)/palonosetron (palo), and olanzapine during the study period.

\section{Discussion}

Recent reports suggest that existing antiemetic guidelines are not well followed, ${ }^{27,28}$ with compliance rates of approximately $50 \%$ to $60 \% .{ }^{29}$ Factors that have been shown to negatively impact guideline adherence include (1) the complexity of prophylactic treatment for HEC, (2) mucositis, (3) depression, (4) polypharmacy, (5) physician workload, and (6) suboptimal communication between provider and patient. ${ }^{27,29-31}$ In one trial, the use of computerized physician order entry for antiemetics resulted in a 97\% rate of compliance with guidelines. ${ }^{32}$ Results from our study shed some light on several issues regarding the clinical use of antiemetics in patients receiving HEC in the United States.

First, our data show that antiemetic agents, including dexamethasone, were used in virtually all patients, which shows that our methodology captured real practice use of these drugs. The frequent use of dexamethasone (in $85 \%-90 \%$ of patients) suggests that clinicians agree with guideline recommendations that steroids should be given routinely to patients receiving HEC. It is likely that comorbid conditions (eg, poorly controlled diabetes) led 


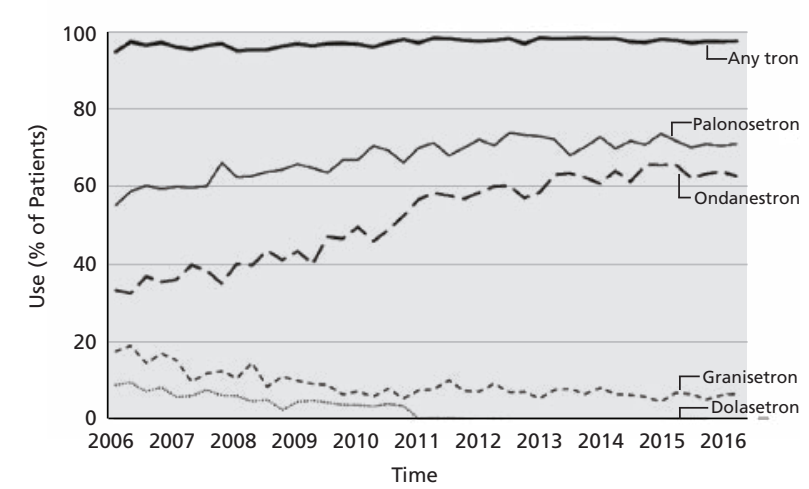

Figure 3. Trends in 5-hydroxytryptamine 3 receptor antagonist (tron) use from 2006 to 2016.

to the omission of this drug in some patients, such that it should not be expected that $100 \%$ would receive it.

The virtually universal receipt of 5HT3RAs in this population is also consistent with guideline recommendations. It is clear from the presented data that palonosetron is a favorite. Other data supporting the common use of palonosetron for HEC in clinical practice come from a recent randomized trial ${ }^{24}$ that accrued patients between August 2014 and March 2015, which evaluated olanzapine versus placebo in conjunction with dexamethasone, a 5HT3RA, and an NK1RA. In this study, clinicians were able to choose which 5HT3RA they used; $76 \%$ chose palonosetron, $24 \%$ chose ondansetron, and $<1 \%$ chose granisetron. It is worth noting that palonosetron is much more expensive than other 5HT3RAs, and that current guidelines support the use of a less expensive 5HT3RA when an NK1RA is also being used. Dolasetron use was infrequent throughout, but

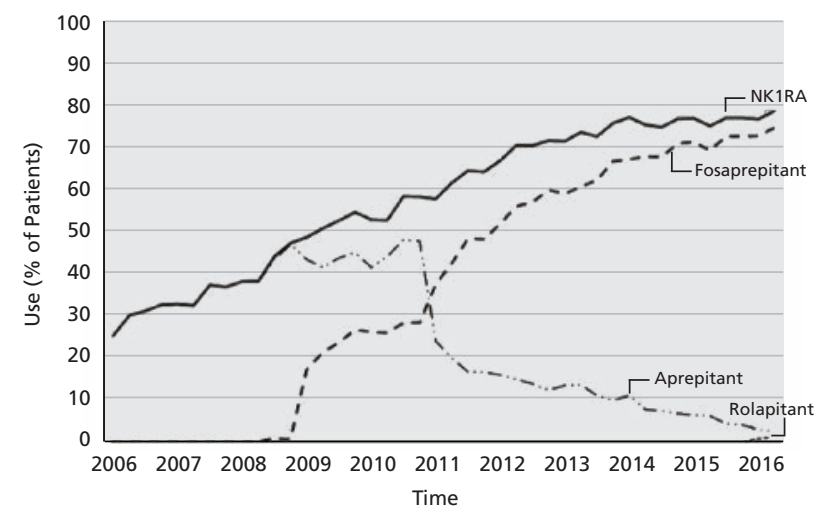

Figure 4. Trends in neurokinin-1 receptor antagonist (NK1RA) use from 2006 to 2016. became substantially more infrequent after 2010. This may have been related to an FDA safety alert in December 2010 regarding a risk of torsades de pointes. ${ }^{33}$

The observation that the total use of one or more 5HT3RAs exceeded 100\%, illustrated in Figure 3, may reflect the fact that patients receiving palonosetron were also given ondansetron scripts to fill in case they experienced nausea or vomiting. However, it could be argued that giving another 5HT3RA to someone who has nausea/vomiting following palonosetron might not be the best approach. Another agent, such as olanzapine ${ }^{34}$ or prochloperazine, ${ }^{35}$ might be a better choice in this situation.

With regard to the use of NK1RAs, the dramatic replacement of aprepitant by fosaprepitant may be due to the more convenient intravenous administration of this NK1RTA, as well as reimbursement issues. It is not clear why, despite the 2014-2015 publications of venous toxicity in a number of patients receiving intravenous fosaprepitant through a peripheral intravenous line,${ }^{12,13}$ there did not appear to be evidence of a subsequent trend away from fosaprepitant and toward aprepitant. As opposed to the almost universal use of guideline-recommended dexamethasone and 5HT3RA use in patients receiving HEC in this study, it is interesting to note that this is not true with the NK1RAs, for which use only recently approached $80 \%$, and in 2011 was only $60 \%$. The NCCN Guidelines for Antiemesis first recommended use of NK1RAs in 2004, and the ASCO guidelines followed suit in 2006.

It is too early to ascertain how the use of the 2 newer NK1RAs, rolapitant and netupitant (presently only available as a combination oral product that also includes palonosetron), will change over time, and whether these new drugs will offer enough of an advantage over fosaprepitant in terms of efficacy, toxicity, and/or cost.

Lastly, the data from this study show that olanzapine was not commonly received, despite the pilot data suggesting its efficacy. ${ }^{21,22,24}$ It will be interesting to see whether it will be used more frequently over the next several years now that antiemetic guidelines include this drug, and the results from a large placebo-controlled, double-blinded clinical trial support its efficacy when combined with an NK1RA, a 5HT3RA, and dexamethasone. . $^{18,19,24}$ 
Trends in Antiemetic Prescribing

\section{Limitations}

The biggest weakness of our study is that it is not clear whether each antiemetic drug was administered as a means of trying to prevent CINV or as a treatment of established CINV. Other limitations include the inability to compare antiemetic use between the academic and community settings, and the lack of regional information. Further, we did not have information regarding doses or costs of the antiemetics used, and it was not possible to be certain whether olanzapine was prescribed for a psychiatric indica- tion or for nausea prophylaxis. Given the limitations of the study design, causation cannot be inferred.

\section{Conclusions}

Our findings showed that dexamethasone and 5HT3RAs were used in most patients receiving HEC, in accordance with guideline recommendations. Less compliance with guidelines was seen with NK1RA use.

\section{References}

1. Basch E, Hesketh PJ, Kris MG, et al. Antiemetics: American Society of Clinical Oncology clinical practice guideline update. J Oncol Pract 2011;7:395-398.

2. Grunberg SM, Deuson RR, Mavros P, et al. Incidence of chemotherapy-induced nausea and emesis after modern antiemetics. Cancer 2004;100:2261-2268.

3. Nasir SS, Schwartzberg LS. Recent advances in preventing chemotherapyinduced nausea and vomiting. Oncology (Williston Park) 2016;30:750-762.

4. Navari RM, Aapro M. Antiemetic prophylaxis for chemotherapy-induced nausea and vomiting. N Engl J Med 2016;374:1356-1367.

5. Kris MG, Gralla RJ, Clark RA, et al. Incidence, course, and severity of delayed nausea and vomiting following the administration of high-dose cisplatin. J Clin Oncol 1985;3:1379-1384.

6. Saito M, Aogi K, Sekine I, et al. Palonosetron plus dexamethasone versus granisetron plus dexamethasone for prevention of nausea and vomiting during chemotherapy: a double-blind, double-dummy, randomised, comparative phase III trial. Lancet Oncol 2009;10:115-124.

7. Aapro MS, Grunberg SM, Manikhas GM, et al. A phase III, double-blind, randomized trial of palonosetron compared with ondansetron in preventing chemotherapy-induced nausea and vomiting following highly emetogenic chemotherapy. Ann Oncol 2006;17:1441-1449.

8. Herrington JD, Jaskiewicz AD, Song J. Randomized, placebo-controlled, pilot study evaluating aprepitant single dose plus palonosetron and dexamethasone for the prevention of acute and delayed chemotherapy-induced nausea and vomiting. Cancer 2008;112:2080-2087.

9. Rapoport BL, Jordan K, Boice JA, et al. Aprepitant for the prevention of chemotherapy-induced nausea and vomiting associated with a broad range of moderately emetogenic chemotherapies and tumor types: a randomized, double-blind study. Support Care Cancer 2010;18:423-431.

10. Grunberg S, Chua D, Maru A, et al. Single-dose fosaprepitant for the prevention of chemotherapy-induced nausea and vomiting associated with cisplatin therapy: randomized, double-blind study protocol-EASE. J Clin Oncol 2011;29:1495-1501.

11. Celio L, Ricchini F, De Braud F. Safety, efficacy, and patient acceptability of single-dose fosaprepitant regimen for the prevention of chemotherapy-induced nausea and vomiting. Patient Pref Adherence 2013;7:391-400.

12. Leal AD, Kadakia KC, Looker S, et al. Fosaprepitant-induced phlebitis: a focus on patients receiving doxorubicin/cyclophosphamide therapy. Support Care Cancer 2014;22:1313-1317.

13. Hegerova LT, Leal AD, Grendahl DC, et al. An analysis of fosaprepitantinduced venous toxicity in patients receiving highly emetogenic chemotherapy. Support Care Cancer 2015;23:55-59.

14. Saito $H$, Yoshizawa $H$, Yoshimori $K$, et al. Efficacy and safety of single-dose fosaprepitant in the prevention of chemotherapy-induced nausea and vomiting in patients receiving high-dose cisplatin: a multicentre, randomised, doubleblind, placebo-controlled phase 3 trial. Ann Oncol 2013;24:1067-1073.

15. Fujii $T$, Nishimura $N$, Kanai $H$, et al. Impact of fosaprepitant use on dermal and vascular adverse events in anthracycline-based regimens administered through peripheral lines [abstract]. J Clin Oncol 2013;31(Suppl):Abstract 9629.

16. Leal AD, Kadakia KC, Looker S, et al. Fosaprepitant-induced phlebitis: a focus on patients receiving doxorubicin/cyclophosphamide therapy. Support Care Cancer 2014;22:1313-1317.

17. Hesketh PJ, Bohlke K, Lyman GH, et al. Antiemetics: American Society of Clinical Oncology focused guideline update. J Clin Oncol 2016;34:381-386.
18. Herrstedt J, Roila F, Warr D, et al. 2016 updated MASCC/ESMO consensus recommendations: prevention of nausea and vomiting following high emetic risk chemotherapy. Support Care Cancer 2017;25:277-288.

19. Ettinger DS, Berger MJ, Aston J, et al. NCCN Clinical Practice Guidelines in Oncology: Antiemesis. Version 2.2017. Accessed February 2, 2018. To view the most recent version of these guidelines, visit NCCN.org.

20. Koth SM, Kolesar J. New options and controversies in the management of chemotherapy-induced nausea and vomiting. Am J Health Syst Pharm 2017;74:812-819.

21. Passik SD, Navari RM, Jung SH, et al. A phase I trial of olanzapine (Zyprexa) for the prevention of delayed emesis in cancer patients: a Hoosier Oncology Group study. Cancer Invest 2004;22:383-388.

22. Navari RM, Einhorn LH, Passik SD, et al. A phase II trial of olanzapine for the prevention of chemotherapy-induced nausea and vomiting: a Hoosier Oncology Group study. Support Care Cancer 2005;13:529-534.

23. Navari RM, Gray SE, Kerr AC. Olanzapine versus aprepitant for the prevention of chemotherapy-induced nausea and vomiting: a randomized phase III trial. J Support Oncol 2011;9:188-195.

24. Navari RM, Qin R, Ruddy KJ, et al. Olanzapine for the prevention of chemotherapy-induced nausea and vomiting. N Engl J Med 2016;375:134-142.

25. Wallace PJ, Shah ND, Dennen T, et al. Optum Labs: building a novel node in the learning health care system. Health Aff (Millwood) 2014;33:1187-1194.

26. Optum. Real World Health care Experiences. Available at: https://www.optum. com/content/dam/optum/resources/productSheets/5302_Data_Assets_Chart_ Sheet_ISPOR.pdf. Accessed February 2, 2018.

27. Caracuel F, Munoz N, Banos U, Ramirez G. Adherence to antiemetic guidelines and control of chemotherapy-induced nausea and vomiting (CINV) in a large hospital. J Oncol Pharm Pract 2015;21:163-169.

28. Tavernier J, Jouannet-Romaszko $M$, Bertucat $H$, et al. Region-wide professional practice evaluation with regards to antiemetic prescription into chemotherapyinduced nausea and vomiting [in French]. Bull Cancer 2016;103:622-631.

29. Davis MP. New therapies for antiemetic prophylaxis for chemotherapy. J Community Support Oncol 2016;14:11-20.

30. Grunberg S, Clark-Snow RA, Koeller J. Chemotherapy-induced nausea and vomiting: contemporary approaches to optimal management. Proceedings from a symposium at the 2008 Multinational Association of Supportive Care in Cancer (MASCC) Annual Meeting. Support Care Cancer 2010;18(Suppl 1):S1-10.

31. Tamura K, Aiba K, Saeki T, et al. Testing the effectiveness of antiemetic guidelines: results of a prospective registry by the CINV Study Group of Japan. Int J Clin Oncol 2015;20:855-865.

32. Kadakia KC, Leal AD, Seisler DK, et al. Antiemetic prescribing practices using a computerized physician order entry system. Support Care Cancer 2014;22:217-223

33. U.S. Department of Health and Human Services. FDA drug safety communication: abnormal heart rhythms associated with use of Anzemet (dolasetron mesylate). Available at: https://www.fda.gov/Drugs/DrugSafety/ ucm237081.htm. Accessed March 1, 2018.

34. Navari RM, Nagy CK, Gray SE. The use of olanzapine versus metoclopramide for the treatment of breakthrough chemotherapy-induced nausea and vomiting in patients receiving highly emetogenic chemotherapy. Support Care Cancer 2013;21:1655-1663.

35. Jones JM, Qin R, Bardia A, et al. Antiemetics for chemotherapy-induced nausea and vomiting occurring despite prophylactic antiemetic therapy. J Palliat Med 2011;14:810-814. 